\title{
Superconductivity of Saturn Rings: Quantum Locking, Rings Disc Thickness and Its Time Creation
}

\author{
Vladimir V. Tchernyi, Andrew Yu Pospelov \\ Modern Science Institute, SAIBR, Moscow, Russia \\ Email: chernyv@bk.ru
}

How to cite this paper: Tchernyi, V.V. and Pospelov, A.Y. (2018) Superconductivity of Saturn Rings: Quantum Locking, Rings Disc Thickness and Its Time Creation. Journal of Modern Physics, 9, 419-432. https://doi.org/10.4236/jmp.2018.93029

Received: December 21, 2017

Accepted: February 8, 2018

Published: February 11, 2018

Copyright $\odot 2018$ by authors and Scientific Research Publishing Inc. This work is licensed under the Creative Commons Attribution International License (CC BY 4.0).

http://creativecommons.org/licenses/by/4.0/

\section{(c) (i) Open Access}

\begin{abstract}
The article demonstrates how rings disc of Saturn was created after appearance of the planetary magnetic field from superconducting iced particles of the protoplanetary cloud moving around planet by chaotic orbits. It is based on the fact that Saturn has magnetic field and the temperature of its vicinity is low enough to have superconductivity. Electromagnetic simulation estimates time of rings disc formation with the thickness about few meters from a few thousand years up to few tens of thousands of years. A rings disk has a stable structure located within magnetic equator of Saturn due to quantum locking of the particles by planetary magnetic field. Also may happened contribution to the rings disc from the debris of the moons migrated inward towards Saturn and particles of the frozen water coming from the geyser of the geologically active satellite (as Enceladus contributed to the E-ring). Suggested mechanism of the rings formation works even in case where only a small fraction of the particles poses superconductivity. Presented electromagnetic modeling of the role of superconducting iced particles of the rings disc origin, dynamics and evolution allow us to enrich the classical theories based on gravitational, mechanical, magnetohydrodynamic and plasma interactions.
\end{abstract}

\section{Keywords}

Origin of Saturn Rings, Origin of Planetary Rings, Superconductivity of the Planetary Rings

\section{Introduction}

Currently, there is no clear understanding of the origin of such a nice structure as Saturn's rings [1]-[11]. As can be seen, the data of the Cassini mission once again confirms the correctness of the H. Alfven's conclusion that the "solar sys- 
tem history as recorded in the Saturn rings structure" [1]. And it becomes to be a physical reality due to applicability of our electromagnetic model to extend common understanding of the Saturn rings origin. It was postulated the ring particles can be collision-generated debris from current moons [2] [3]. With this approach, there are two ideas proposed for today: massive asteroid in the Roche zone was destroyed by a passing Saturn or Titan-sized satellite as it migrates inward towards Saturn; a massive object was tidally disrupted during a close passage by Saturn. But these theories do not allow a single common position to use the same physical phenomenon in order explain many experimentally observed effects in the rings. There was no yet clear explanation why and how chaotic orbits of the particles rotating around Saturn coming to the equator rings disk and holding inside of it for a long time. There was no yet even one theory allow us to calculate and estimate thickness of the rings disk and time of the rings disk formation from the iced particles of the protoplanetary cloud.

An interesting idea of the rings formation comes from the similarity of the picture of the Saturn rings to the picture when iron particles creates dense and rarefied regions in a nonuniform magnetic field near by the magnet on laboratory table (please, see Figure 1).

The question is how we can get such a good looking structure for the rings of Saturn located within equator plane from the situation when iced particles moving around Saturn by chaotic orbits within protoplanetary cloud.

Recently, Cassini mission has demonstrated that the rings consist of pure ice, 93\% with some carbon matter impurities, 7\%

(https://www.nasa.gov/mission_pages/cassini/media/cassini-090204.html). The temperature in the area of the rings is about 70-110 K. That's why superconducting substance may be possible in space behind the belt of asteroids. It is demonstrated in [11]-[35] that rings disc is result of interaction of superconducting carbon doped ice particles of the protoplanetary cloud with the nonuniform

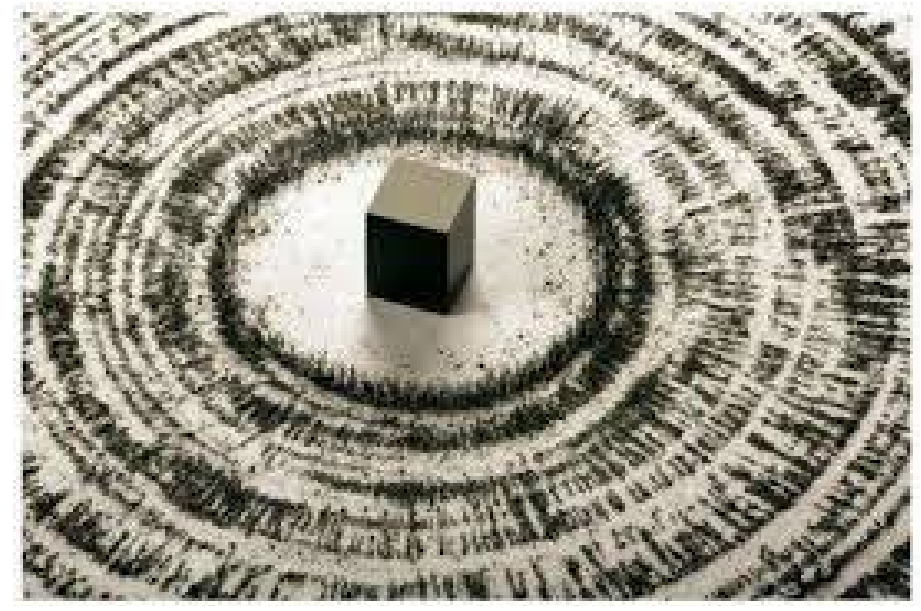

Figure 1. Iron particles creates dense and rarefied regions in nonuniform magnetic field near by the magnet on laboratory table. $\underline{\text { https://wadevenden.files.wordpress.com/2014/07/magnet.jpg?w=500 }}$ 
magnetic field of Saturn. Due to Meissner phenomenon, particles with chaotic orbits go to magnetic equator plane where there is a minimum of magnetic energy. The high-temperature superconductivity was discovered by J. G. Bednorz and K. A. Muller in 1986 [36]. G. V. Babushkina et al. presented superconductivity of ice in 1986 [37] and M. Cote et al. made it for C36 in 1998 [38]. Surprisingly, assuming superconductivity of the Saturn rings matter, many difficulties of the rings origin can find a simple explanation:

- origin, evolution, and dynamics of the rings;

- considerable flattening and the sharp edges of the ring system;

- why particles of the rings are separated;

- formation of "spokes" in the ring B;

- high radio-wave reflectivity and low brightness of the rings;

- anomalous reflection of circularly polarized microwaves;

- spectral anomalies of thermal radiation of the rings;

- why planetary rings in the solar system appear only after asteroid belt;

- why the Earth has no rings.

\section{Quantum Phenomena of Superconductor Related to the Saturn Rings Origin}

Ice is complicated matter. There is no enough knowledge about space ice. But as we can see it below an important fact is that even small amount superconducting matter may hold more than 70,000 times its own weight [39]. The experimental set up of how high temperature superconductivity is working in laboratory B. Almog presented in Chicago at the ASTC Conference in 2011 (G. Deutscher et al., [39]). It is based on the discovery of a fluxon by A. Abrikosov (Abrikosov vortex) and research of A. Tonomura, Hitachi [40] [41] who for the first time demonstrated quantum nature of superconductivity using the electronic microscope with high resolution. It was demonstrated experimentally that superconductivity has quantum nature and the magnetic field is quantized inside the superconductor.

Let's discuss result of [39] [40] and [41]. In some situation magnetic fields lines strands of the magnetic field remain inside superconductor, it gets trapped inside superconductor (Figure 2).

Because interaction of superconductor with external magnetic field is a quantum physical phenomenon the magnetic field inside superconductor behaves like a quantum particle, like a quantum object [39] [40] and [41]. And as a fact magnetic field is magnetized inside superconductor (please, see it on Figure 3). Some of the flux line becomes to be pin, and they never could take move. The reason for that is that superconductor doesn't like magnetic field lines moving around. What it actually does, it locks them in place. By doing that, it blocks itself.

Why it so? Under the low temperature some of magnetic lines will be expelled outside of the sample of superconductor because of Meissner phenomenon, and at the same time some of them will remain inside the sample and superconductor locks this magnetic field flux lines inside itself (please, see Figure 4 [39]). 


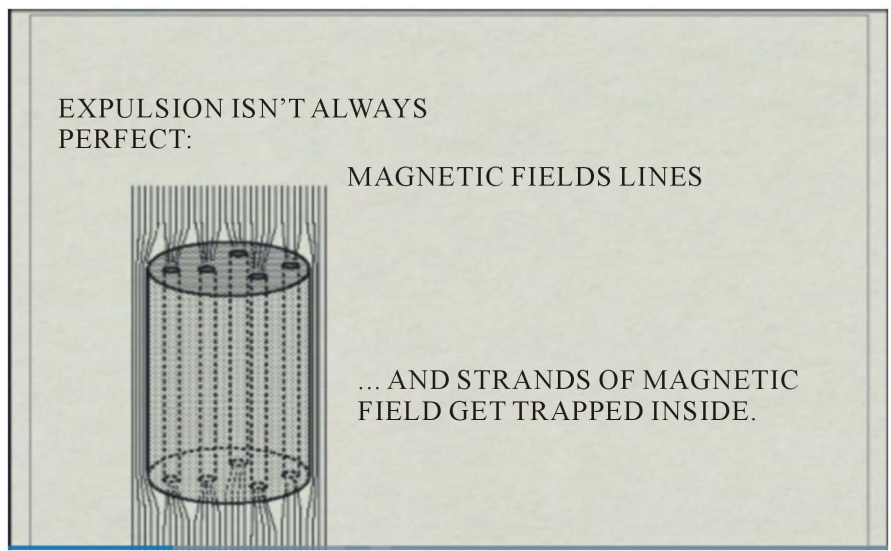

Figure 2. Magnetic fields lines strands of the magnetic field remain inside superconductor; it gets trapped inside superconductor [39] [40] and [41].

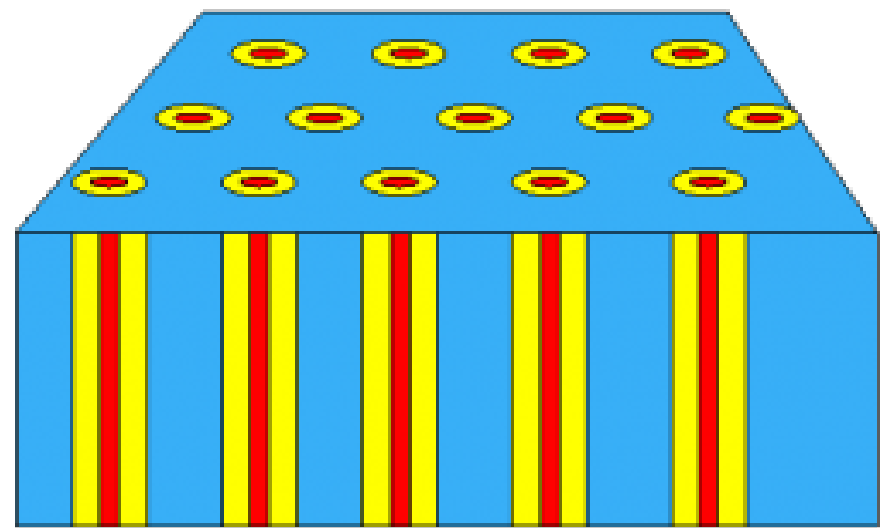

Figure 3. http://makeitquantum.ru/superconductor-in-magnetic-field/. Magnetic field is magnetized inside superconductor. Some of the flux line becomes to be pin, they don't move [39] [40]. This type superconductor allows only partial expulsion of a magnetic field. The volume of it is permeated with a kind of strands inside, also known as "Abrikosov vortices" [41], where the superconductor becomes a normal conductor.

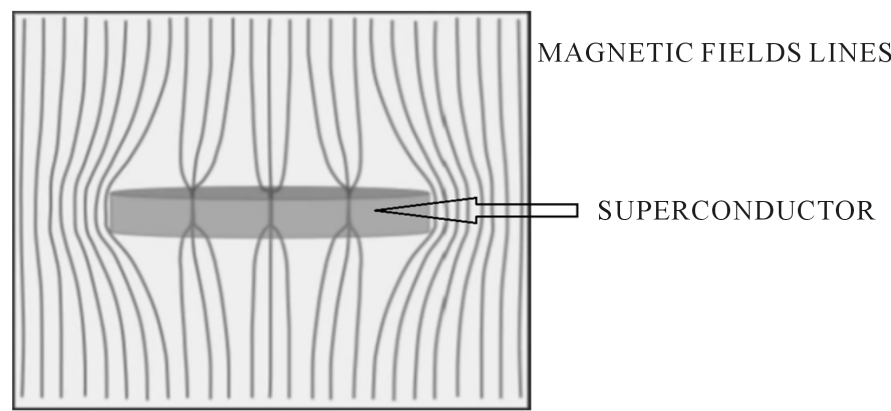

Figure 4. Under the low temperature some of magnetic lines will be expelled outside of the sample of superconductor because of Meissner phenomenon. And at the same time some of them will remain inside the sample and superconductor locks this magnetic field flux lines inside itself [39]. 
The action of "locking" prevents the disc from the moving in space. It is quantum locking, quantum levitation.

The biggest part of the sample of matter in experimental demonstration [39] is not a superconductor (Figure 5). Superconductor is only small part of the sample. At the same time it was shown that the thin superconductor is able to hold more than 70,000 times of its weight. So, it means that we do have a very strong phenomenon of the quantum locking of the superconductor.

In case of Saturn rings we do have situation which looks similar to the case demonstrated above on the bases of research has been done by G. Deutscher, $M$. Azoulay, B. Almog, and B. Deutscher [39]. The rings particles contain 93\% of ice and only $7 \%$ of carbon. So, it means that even if ice of the rings particles is not completely superconducting but phenomenon of superconductivity will be responsible for the rings disk formation due to contains only $7 \%$ of carbon. And consequently superconducting rings iced particles will be strong enough trapped into the magnetic equator plane by the Saturn magnetic field due to phenomena of quantum locking and quantum levitation. The rings disk will be suppressed by the magnetic pressure from both sides along the $z$ axis.

\section{Electromagnetic Simulation of the Origin of the Rings Disc of Saturn}

Following our electromagnetic model prior emergence of the Saturn magnetic field all the particles within the protoplanetary cloud are located on the chaotic Keplerian orbits, exhibiting a balance of the force of gravity and the centrifugal force. With emergence of the Saturn magnetic field, the superconducting particles of the protoplanetary cloud begin to demonstrate an ideal diamagnetism (Meissner-Ochsenfeld phenomenon). Particles begin to interact with the magnetic field and all the particles become to be involved in an additional azimuth-orbital motion. We try to estimate the result of this motion. We will follow

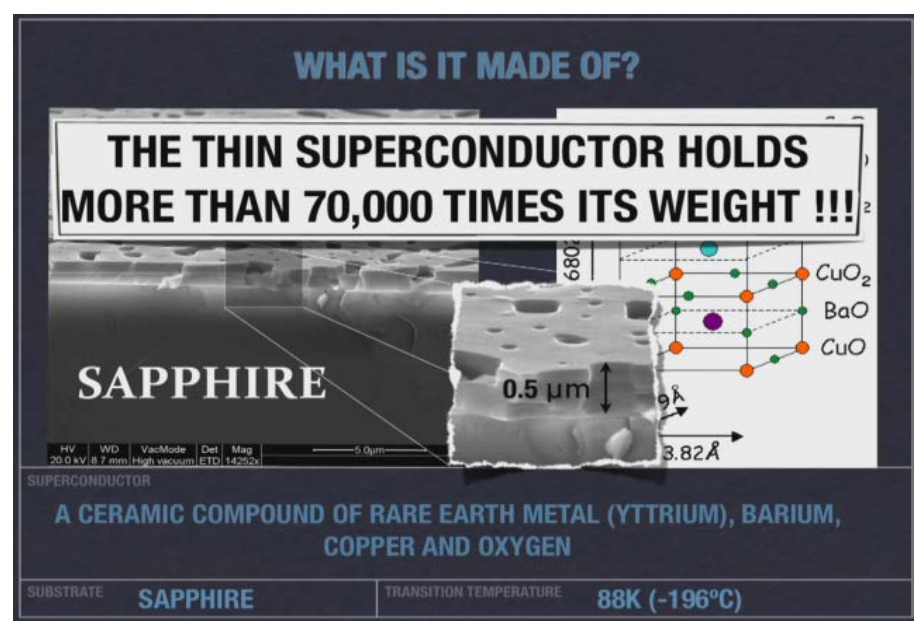

Figure 5. The sample of matter used for the demonstration [39]. The biggest part of the sample of material in experimental demonstration of is not a superconductor. 
procedure of electromagnetic modeling already demonstrated earlier [21] [22] [23] [24] [25]. Let's consider diamagnetic ball with mass $m$ flying in a gravitational and magnetic field of a planet with a given initial velocity and distance from the center of the planet. Find its trajectory. In the spherical coordinate system we get:

$$
r=\sqrt{x^{2}+y^{2}+z^{2}}, \varphi=\arctan (y / x), \theta=\arccos (z / r)
$$

And the acceleration vector in this coordinate system consequently is

$$
\begin{aligned}
\boldsymbol{a}= & \boldsymbol{e}_{r}\left(\ddot{r}-r \dot{\theta}^{2}-r \dot{\varphi}^{2} \sin ^{2} \theta\right)+\boldsymbol{e}_{\theta}\left(r \ddot{\theta}+2 \dot{r} \dot{\theta}-r \dot{\varphi}^{2} \sin \theta \cos \theta\right) \\
& +\boldsymbol{e}_{\varphi}(r \ddot{\varphi} \sin \theta+2 \dot{r} \dot{\varphi} \sin \theta+2 r \dot{\theta} \dot{\varphi} \cos \theta)
\end{aligned}
$$

Velocity is $\boldsymbol{v}=\dot{\boldsymbol{r}} \boldsymbol{e}_{r}+r \dot{\theta} \boldsymbol{e}_{\theta}+r \dot{\varphi} \sin \theta \boldsymbol{e}_{\varphi}$ and $\boldsymbol{r}=r \boldsymbol{e}_{r}$

The magnetic induction of a planet is

$$
\boldsymbol{B}=\frac{\mu_{0}}{4 \pi} \frac{3 \boldsymbol{r}(\boldsymbol{\mu r})-\boldsymbol{\mu} r^{2}}{r^{5}}
$$

The magnetic field is $\boldsymbol{B}=\mu_{0} \boldsymbol{H}$, and magnetization $\boldsymbol{B}=\mu_{0}(\boldsymbol{H}+\boldsymbol{M})$, $\boldsymbol{M}=\chi_{m} \boldsymbol{H}$.

We place now a diamagnetic sphere into the uniform magnetic field and see what happens.

No currents flow, then $\nabla \times \boldsymbol{H}=0$, and we introduce a scalar potential $\boldsymbol{H}=-\nabla \psi \quad \operatorname{div} \boldsymbol{B}=0$, then $\nabla^{2} \psi=0$. A uniform magnetic field is represented by its magnetic potential

$$
\begin{gathered}
\psi_{0}=-H_{0} z=-H_{0} r \cos \theta \\
\psi= \begin{cases}-H_{0} r \cos \theta+\frac{a \cos \theta}{r^{2}}, & r>R \\
-b r \cos \theta, & r<R\end{cases} \\
a=\frac{1}{3} R^{3} M, b=H_{0}-\frac{1}{3} M
\end{gathered}
$$

Here $b$ is the value for the uniform magnetic field inside the particle: $M=\chi_{m} H_{\mathrm{int}}=\chi_{m} b$. For a superconductor, we get $\chi_{m}=-1$.

So, finally an effective magnetic dipole moment is given $\boldsymbol{m}=-2 \pi R^{3} \boldsymbol{H}_{0}$.

The potential energy of a magnetic dipole $\boldsymbol{m}$ is $U=-\boldsymbol{m} \boldsymbol{B}$. For a rigid dipole, the force then is

$\boldsymbol{F}=(\boldsymbol{m} \nabla) \boldsymbol{B}$, then we obtain $\boldsymbol{F}=-\pi \mu_{0} R^{3} \nabla H_{0}^{2}$.

In a simplified version, a magnetic moment for the particle in CGS units is $\boldsymbol{M}=-R^{3} \boldsymbol{H}$.

We place the origin of the coordinates at the center of a planet, and the $z$ axis direct along the magnetic moment of a planet (orthogonal to equator). Then the magnetic energy will become as [21] [22] [23] [24] [25]:

$$
U=-\mu_{p} \boldsymbol{B}=R^{3} H^{2}=\frac{R^{3} \mu^{2}}{r^{6}}\left(3 \cos ^{2} \theta+1\right)
$$

Here $\theta$-the angle between the vector $\boldsymbol{r}$ and the $z$ axis. It can be seen from 
this expression, that the magnetic energy of the superconducting particle has a minimum value when the radius-vector $\boldsymbol{r}$ is in a plane of the magnetic equator plane, $(\cos \theta=0)$ [11] [24]-[35]. It means that eventually particle during an interaction with magnetic field of planet tries to get position within magnetic equator plane and then it continues move around Saturn within the rings disk in accordance with Kepler's Law.

Now going to spherical coordinates we get

$$
\nabla U=\frac{\partial U}{\partial r} \boldsymbol{e}_{r}+\frac{1}{r} \frac{\partial U}{\partial \theta} \boldsymbol{e}_{\theta}
$$

The Newton equation of motion is then

$$
m \boldsymbol{a}=-\left(\frac{G m M}{r^{2}}+\frac{\partial U}{\partial r}\right) \boldsymbol{e}_{r}-\frac{1}{r} \frac{\partial U}{\partial \theta} \boldsymbol{e}_{\theta}
$$

In order to do calculations we take follow values for parameters: at equator $R_{S}=60268 \mathrm{~km}, M=5.6846 \times 10^{26} \mathrm{~kg}, \quad G=6.67259 \times 10^{-11} \mathrm{~m}^{3} \cdot \mathrm{kg}^{-1} \cdot \mathrm{s}^{-2}$ and the density of an ice particle $\rho \approx 1 \Gamma / \mathrm{cm}^{3}$ The Saturn magnetic field at the equator is 0.2 gauss. Then the Saturn magnetic moment may be calculated as

$$
B=\frac{\mu_{0}}{4 \pi} \frac{\mu}{R_{S}^{3}}, \mu=\frac{4 \pi R_{S}^{3} B_{S}}{\mu_{0}}, \mu=4.378 \times 10^{25} \mathrm{~m}^{2} \cdot A
$$

Eventually, calculations give us time for particle to change the orbit to move into magnetic equator plane due to additional magnetic force of Meissner phenomenon is from a few thousand years up to few tens of thousands of years. So, during this time the additional magnetic force will modify significantly the orbit of the particle.

To get thickness of the rings sombrero we consider a superconducting rings disc around a planet and evaluate a magnetic field around. Estimation of the sombrero rings thickness gives us about 10 meters. This result is coincident with experimental data.

\section{Discussion of Some Detail Related to the Estimation of the Rings Disc Time Formation}

For a more correct estimation of the time formation of the rings disk other important factors should be taken into account. It is clear particle's trajectory under influence of Meissner phenomenon in the azimuth-orbital direction may be disturbed due to additional magnetic force because magnetic field of Saturn is nonuniform. It may increase the orbit of particle and time to come to magnetic equator plane will be even more. Also, we made simulation for one particle. However, there is a significant amount of particles filling the protoplanetary cloud, and collisions between particles during the process of formation of the Saturn rings will increase their azimuth-orbital movements, and the time for particles to come together within magnetic equator plane will be much larger.

It's important to remember that orbital resonances, moons and the ring particles as well as mechanical, plasma and magnetohydrodynamic interactions can 
play an important role in forming a specific structure of the rings and gaps please, see [2] [3] and Ref. [11]-[35]. And these factors also may contribute to the time of the considering process of the dynamic and evolution of the initial origin of existing structure of the Saturn rings.

Also there is probability for some other mechanism to participate in the process of dynamic and evolution of the rings disk. During the time a contribution may happen to the matter of the rings disc of the particles from the debris of the moon migrated inward towards Saturn [2] [3]. Also, another situation may happen when particles of the frozen water coming from the geyser of the geologically active satellite go to the rings disc due to magnetic coupling between Saturn and its sputnik (Figure 6).

An important discovery was made in 2005. The Cassini spacecraft started multiple close flybys of Enceladus, revealing its surface and environment in greater detail. In particular, Cassini discovered water-rich plumes venting from the south polar region [42]. Cryovolcanoes near the south pole shoot geyser-like jets of water vapor up to $500 \mathrm{~km}$, molecular hydrogen, other volatiles, and solid material, including sodium chloride crystals and ice particles, into space, totaling about $200 \mathrm{~kg}$ (440 lb) per second [43]. Over 100 geysers have been identified [44]. Some of the water vapor falls back as "snow"; the rest escapes, and supplies most of the material making up Saturn's E ring [45] [46] (Figure 7). The intensity of the geysers of Enceladus depends on how close or far the satellite is from Saturn [47].

Certainly process of supplying particles to the rings disc from the geyser of the satellite of Saturn could not continue forever. It works only during the time of the geological activity of the satellite. It may happen at any moment of the long time existence of the Saturn system. This conclusion compels us to refer to the

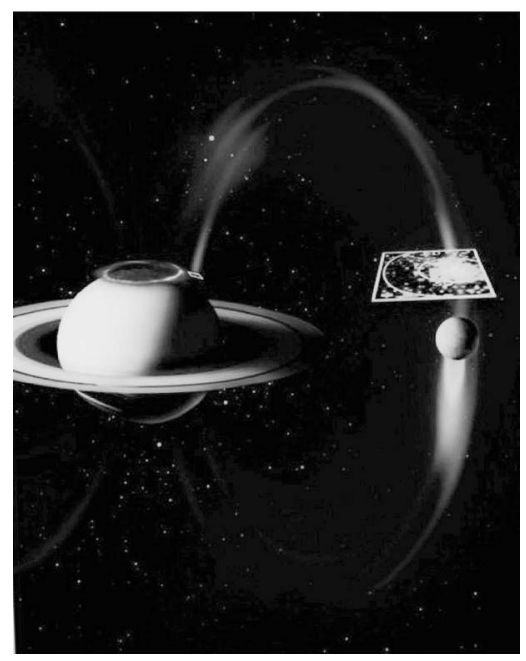

Figure 6. Image and Title Credit: NASA/JPL/JHUAPL/University of Colorado/Central Arizona College/SSI. Electrical circuits between Saturn and Enceladus. The magnetic field also provides a path for electrons to flow as illustrated in the graphic above. There is a magnetic coupling between Saturn and Enceladus. PIA13765. April 20, 2011.

https://saturn.jpl.nasa.gov/resources/5289/ 


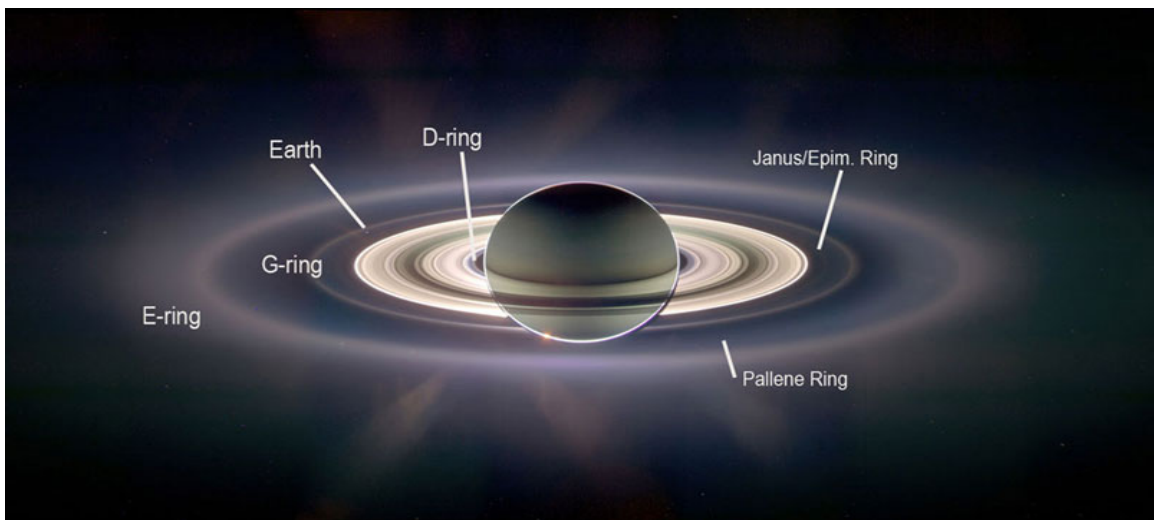

Figure 7. Image and Title Credit: NASA/JPL/JHUAPL/University of Colorado/Central Arizona College/SSI. Saturn rings system with E ring. Water vapor from the geysers of Enceladus supplies most of the particles making up an external Saturn's E ring. This process goes in accordance with magnetic coupling between the Saturn and Enceladus as it shown on the Figure 6. https://saturn.jpl.nasa.gov/resources/

result previously obtained for the B ring by $\mathrm{M}$. Hedman and P. Nicholson in 2016 [48]. We have received a totally new understanding of what is the matter of the B ring of Saturn.

After studying photos of the planet taken by the Cassini spacecraft, the experts came to the conclusion that the most striking B ring of Saturn's demonstrates for the observer an optical illusion. The mass of the B ring is only from one-half to one-seventh of the previously existing assessment. Those measurements reveal that the $\mathrm{B}$ ring, which makes up 80 percent of the total ring mass, is about 0.4 times that of Saturn's moon Mimas. In fact, B ring of Saturn contain three times less matter than previously thought. "The best analogy of this situation with B ring of Saturn is something like a fog over the meadow may seem less transparent and empty, than a water-filled pool, which has a much higher density than the fog" (P. Nicholson [49]). Because of small particles size of B ring might be related to earliest day of the Saturn system or it also could mean that in the formation of B ring at some time ago there is a possibility of participation of geysers of another moon of Saturn just as it does with the E ring.

Following our electromagnetic model of the origin of Saturn rings scenario for $\mathrm{B}$ ring may be presented as follows. Initially superconducting particles of the protoplanetary cloud after appearance of the Saturn magnetic field went to the magnetic equator plane and formatted a rings disc including B ring. The magnetic well of the superconducting rings disk could attract inside itself additional particles of the debris of destroyed moon approaching to the Saturn as well as particles coming from the geyser of another satellite. Because particles of ice following from debris of the moon or the frozen water erupted with geyser also may poses diamagnetism and superconductivity.

How the rings formed remains a mystery. The best guess of L. Esposito is that a single moon about half the mass of Mimas was ripped up around 200 million years ago [49]. Using all the measurements from Cassini's dust-counting in- 
strument since the spacecraft arrived at Saturn in 2004, Kempf and colleagues showed that the still-bright rings collect too much dust pollution to have maintained their youthful shine for billions of years [4] [49].

At the same time, following last two facts we can conclude that it does not mean that most of the rings disc particles are not relicts of the earliest day of Saturn system formation. The best description of the situation with the ring origin and age is given by the title of the paper written by P. Estrada, R. H. Durisen and J.N. Cuzzi: "After the Cassini grand finale, is there a final consensus on ring origin and age?" [50]

All experimental data of Cassini we discussed above it is confirmation that most particles of the Saturn rings are relicts of the earliest day of Saturn system formation. Certainly, the ring we see today is not the same completely at the beginning of it appearance. Formation of the rings disc it is not a one-off event. The process of existence of the rings disk has a long history started from it dynamics of the initial origin from the particles of the protoplanetary cloud and it continuing up today. The Saturn system might go through cycles of moons and rings and data from Cassini spacecraft show that the gas giant didn't always have its iconic icy band [49].

\section{Conclusions}

Recently, we coined the hypotheses that the ice matter of the Saturn rings particles or part of it may be superconducting. The above consideration has demonstrated once again how Saturn's rings initially could have formed from the superconducting icy particles of the protoplanetary cloud and in addition also with some other mechanisms of the observing phenomena. The most intriguing part of this process of the Saturn rings origin is electromagnetic interaction of superconducting iced particles with magnetic field of Saturn.

Saturn rings particles are relicts of the earliest day of the Saturn system formation. And rings disc was formatted after appearance of the magnetic field of planet in accordance with presented above scenario. Due to the action of the additional magnetic force of the Meissner phenomenon, all chaotic orbits of the particles eventually moved from the protoplanetary cloud to magnetic equator plane where there is a minimum of magnetic energy. Nearby Saturn, the sphere has a uniform magnetization. Particles within the rings disc become to be blocked by quantum locking and suppressed by external magnetic field. Magnetic well of the rings disc is sufficiently disturbing picture of Saturn magnetic field in the area of equator. If some other superconducting particle is flying nearby the rings disc, then the magnetic well will eventually attract the particle within the disc and trapped them inside.

So, the time of initial formation of the rings disc system with thickness of a few meters due to electromagnetic interaction of superconducting iced particles of the protoplanetary cloud with magnetic field of Saturn may be estimated as from a few thousand of years up to few tens of thousands of years. And this is 
the time of the origin, dynamics and formation of the initial state of the system of Saturn rings after appearance of the magnetic field of Saturn. During this time, the chaotic orbits of the superconducting iced particles of the protoplanetary cloud which are moving around Saturn will collapse into the disc of rings.

At the same time, due to a long time existence of the Saturn rings, the other processes also need to be considered which could contribute to the dynamics and evolution of the rings disk. It is necessary to underline that some other sudden event like moon coming to the Saturn and becoming to be destroyed to the debris or water eruption by the geyser of the satellite of Saturn, all of them could contribute to the dynamic and evolution of the existing structure of the rings disc matter. For example, some of the water vapor by the geyser of Enceladus falls back as "snow"; the rest escapes, and supplies most of the material making up Saturn's E ring. We can imagine the same situation could happen earlier with another satellite and $\mathrm{B}$ ring.

It is interesting feature that suggested mechanism of the initial formation of the rings due to electromagnetic interaction works even in case when only small fraction of the iced particles poses superconductivity. This conclusion is based on result of laboratory experiment when the small scale frozen superconductor located on the big enough size of substrate is trapped in the surrounding magnetic field and it is able to hold much more times its weight. Magnetic field is magnetized inside the superconductor. Some of the flux line becomes to be pin, they don't move. And superconductor blocks itself within magnetic field. It happened because superconductivity has quantum nature.

In fact, orbital resonances between satellites, moons and ring particles play an important role in forming a specific structure of the rings system gaps and enhancing the influence of the satellites as well as the gravitational, mechanical, magnetohydrodynamic, dusty plasma and gas-plasma, interactions.

It is a pleasing surprise, how many features of the Saturn rings our model can explain. If applied to other systems, it has the potential to enrich our understanding of the space natural laboratory in general.

\section{References}

[1] Alfven, H. (1968) Icarus, 8, 75-81. https://doi.org/10.1016/0019-1035(68)90064-X

[2] Fridman, A.M. and Gorkavyi, N.N. (1999) Physics of Planetary Rings: Celestial Mechanics of a Continuous Media. Springer-Verlag, Berlin Heidelberg. https://doi.org/10.1007/978-3-662-03918-2

[3] Canup, R.M. (2010) Nature, 468, 943-946. https://doi.org/10.1038/nature09661

[4] Kempf, S., et al. (2017) The Age of Saturn's Rings Constrained by the Meteoroid Flux into the System. American Geophysical Union Meeting, New Orleans, 13 December 2017.

[5] Henry, J. (2006) Journal of Creation, 20, 123. http://www.creationconcepts.org/resources/RINGS.pdf

[6] Rowan, L., Sanchez-Lavega, A., Gombosi, T.I., Hansen, K.S., Porco, C.C., et al. 
(2005) Science, 307, 1222-1276.

https://doi.org/10.1126/science.307.5713.1222

[7] Burns, J.A. and Cuzzi, J.N. (2006) Science, 312, 1753-1755.

https://doi.org/10.1126/science.1114856

[8] Dougherty, M., Esposito, L. and Krimigis, T. (2009) Saturn from Cassini-Huygens. Springer, Dordrecht. https://doi.org/10.1007/978-1-4020-9217-6

[9] Cuzzi, J.N., Burns, J.A., Charnoz, S., Clark, R.N., Colwell, J.E., Dones, L., Esposito, L.W., Filacchione, G., French, R.G., Hedman, M.M., Kempf, S., Marouf, E.A., Murray, C.D., Nicholson, P.D., Porco, C.C., Schmidt, J., Showalter, M.R., Spilker, L.J., Spitale, J.N., Srama, R., Sremčević, M., Tiscareno, M.S. and Weiss, J. (2010) Science, 327, 1470-1475. https://doi.org/10.1126/science.1179118

[10] Tiscareno, M.S., Colin, J., Mitchell, C.S., Murray, C.D., Di Nino, D., Hedman, M.M., Schmidt, J., Burns, J.A., Cuzzi, J.N., Porco, C.C., Beurle, K. and Evans, M.W. (2013) Science, 340, 460-464. https://doi.org/10.1126/science.1233524

[11] Tchernyi, V.V. and Pospelov, A.Yu. (2015) International Journal of Modern Physics and Application, 2, 65-72. http://www.aascit.org/journal/ijmpa

[12] Pospelov, A.Yu. and Tchernyi, V.V. (1995) Electromagnetic Properties Material Forecast in the Planet Rings by the Methods of Functionally Physical Analysis. Proceedings of International Scientific-Methodological Conference on Innovative Design in Education, Techniques and Technologies, Volgograd, 1995, 75-77.

[13] Pospelov, A.Yu., Tchernyi, V.V. and Girich, S.V. (1998) Planet's Rings: Super-Diamagnetic Model and New Course of Investigations. Proceedings of SPIE 42nd Annual Meet, San Diego, 27 July-1 August 1997, Vol. 3116, 117-128.

[14] Pospelov, A.Yu., Tchernyi, V.V. and Girich, S.V. (1998) Possible Explanation of the Planet's Rings Behavior in the Radio and MM-Wave Range via Superdiamagnetic Model. SPIE International Symposium on Astronomical Telescopes and Instrumentation, Kona, 20-23 July 1998, Paper No. 73.

[15] Pospelov, A.Yu., Tchernyi, V.V. and Girich, S.V. (1998) Superdiamagnetic Model of Planetary Rings Behavior in the Millimeter and Submullimeter Range. Digest 3465-4th International Conference on MM and SMM Waves and Applications, Proceedings of SPIE 43th Annual International Symposium, San Diego, 20-23 July 1998, 172-173.

[16] Girich, S.V., Pospelov, A.Yu. and Tchernyi, V.V. (1998) Radar Data Explanation via Superdiamagnetic Model of the Saturn's Rings. Annual Report of AAS, 30 th Meeting Division of Planetary Science, Madison, 11-16 October 1998, 1043.

[17] Tchernyi, V.V., Pospelov, A.Yu. and Girich, S.G. (1998) Studies on the Rings of Saturn. The Academy for Future Science, Los Gatos.

[18] Pospelov, A.Yu., Tchernyi, V.V. and Girich, S.G. (1999) Anomalous Inversion of Polarization of Icy Satellites and Saturn's Rings: Superdiamagnetic Model. Proceedings of 44th SPIE Annual Meeting, Denver, 18-23 July 1999, Vol. 3754, 329-333.

[19] Pospelov, A.Yu., Tchernyi, V.V. and Girich, S.G. (2000) Are Saturn's Rings Superconducting? Progress in Electromagnetic Research Symposium, Cambridge, 5-14 July 2000, 1158.

[20] Pospelov, A.Yu., Tchernyi, V.V. and Girich, S.G. (1999) Are Saturn Rings Superconducting? University of Alabama, Huntsville, NASA Marshall Space Flight Center, Huntsville Space Physics Colloquium.

[21] Tchernyi, V.V. and Pospelov, A.Yu. (2005) Progress in Electromagnetic Research, 
52, 277-299.

[22] Tchernyi, V.V. and Pospelov, A.Yu. (2005) Fizika Volnovykh Processov I Radiotehnicheskih System, 8, 4-16. (In Russian)

[23] Tchernyi, V.V. and Pospelov, A.Yu. (2007) Astrophysics and Space Science, 307, 347-356.

[24] Tchernyi, V.V. and Chensky, E.V. (2005) Journal of Electromagnetic Waves and Applications, 19, 1997-2006.

[25] Tchernyi, V.V. and Chensky, E.V. (2005) Geoscience and Remote Sensing Letters, 2, 445-446.

[26] Tchernyi, V.V. (2002) Possible Superconductivity of Saturn Rings. University of Hawaii, Institute for Astronomy, Honolulu.

[27] Tchernyi, V.V. (2006) Prikladnaya Fizika (Applied Physics), 5, 10-16. (In Russian)

[28] Tchernyi, V.V. (2006) Possible Role of Superconductivity and Electromagnetism for the Origin of the Rings of Saturn. Fundamental Principles of Engineering Sciences, Moscow, 25-27 October 2006, 257-259. (In Russian)

[29] Tchernyi, V.V. (2009) Origin of the Saturn Rings: Electromagnetic Model of the Sombrero Rings Formation. In: Denis, J.H. and Aldridge, P.D., Eds., Space Exploration Research, Nova Science Publishers, New York, Chapter 11, 261-275.

[30] Tchernyi, V.V. (2010) Journal of Magnetohydrodynamics, Plasma and Space Research, 14, 385-398.

[31] Tchernyi, V.V. (2009) To Discovery of Initial Formation (Origin) of the Sombrero Rings of Saturn: Role of Electromagnetism. International Astronomical Union, Assembly, 27 th General Assembly, Rio de Janeiro, 3-14 August 2009, Report No. 263, 56-63.

http://www.observatorio.unal.edu.co/investigacion/archivos/AbsBookXXVIIga09.pdf

[32] Tchernyi, V.V. (2009) To Discovery of the Saturn Rings Origin: Possible Separation and Collision of the Saturn Rings Particles during Its Evolution Based on Superconductivity and Electromagnetism. International Astronomical Union, Assembly, 27 th General Assembly, Rio de Janeiro, 3-14 August 2009, Report No. 263, 63-64.

[33] Tchernyi, V.V. (2013) Journal of Modern Physics, 4, 17-23.

[34] Cherny, V.V. (2013) American Journal Astronomy and Astrophysics, 1, 15-22.

[35] Tchernyi, V. (2013) International Journal of Astronomy and Astrophysics, 3, 412-420. https://doi.org/10.4236/ijaa.2013.34049

[36] Bednorz, J.G. and Müller, K.A. (1986) Zeitschrift für Physik B, 64, 189-193.

[37] Babushkina, G.V., Kobelev, L.Ya., Yakovlev, E.N. and Babushkin, A.N. (1986) Physics of Solid State, 28, 3732-3734. (In Russian)

[38] Côté, M., Grossman, J.C., Cohen, M.L. and Louie, S.G. (1998) Physical Review Letters, 81, 697.

[39] Deutscher, G., Azoulay, M., Almog, B. and Deutscher, B. (2011) Quantum Levitation, Quantum Locking, Quantum Trapping. ASTC Conference, Chicago, 15-18 October 2011.

http://library.fora.tv/2012/10/25/Dr_Boaz_Almog_Quantum_Levitation

[40] Tonomura, A., et al. (2001) Nature, 412, 620-622.

[41] Olson, C.J., Reichhardt, C. and Nori, F. (1998) Physical Review Letters, 81, 3757.

[42] Hansen, C.J., Esposito, L., et al. (2006) Science, 311, 1422.

[43] Spencer, J.R. and Nimmo, F. (2013) Annual Review of Earth and Planetary Sciences, 
41, 693-717.

[44] Dyches, P., Brown, D., et al. (2014) Cassini Spacecraft Reveals 101 Geysers and More on Icy Saturn Moon. NASA, Washington DC.

[45] NASA News (2015) Icy Tendrils Reaching into Saturn Ring Traced to Their Source.

[46] NASA (2006) Ghostly Fingers of Enceladus. NASA/JPL/Space Science Institute.

[47] Meyer, J. and Wisdom, J. (2007) Icarus, 188, 535-539.

https://doi.org/10.1016/j.icarus.2007.03.001

[48] Hedman, M.M. and Nicholson, P.D. (2016) Icarus, 279, 109-124.

http://dx.doi.org/10.1016/j.icarus.2016.01.007

[49] Grossman, L. (2018) Science News, 193, 7.

[50] Estrada, P., Durisen, R.H. and Cuzzi, J.N. (2017) Ballistic Transport: After the Cassini Grand Finale, Is There a Final Consensus on Ring Origin and Age? American Geophysical Union Meeting, New Orleans, 12 December 2017. 